\title{
Functional PAN-based Monoliths with Hierarchical Structure for Heavy Metal Removal
}

Gang Wang, Jianren Wang, Hao Zhang, Fei Zhan, Tingting Wu, Qidi Ren and Jieshan

$$
\text { Qiu }^{*}
$$

State Key Lab of Fine Chemicals, Liaoning Key Lab for Energy Materials and Chemical Engineering, PSU-DUT Joint Center for Energy Research, School of Chemical Engineering, Dalian University of Technology, Dalian 116024, China.

\section{Abstract:}

Efficient removal of lead from aqueous media remains a serious task for public health and environment. Here polyacrylonitrile (PAN) monolith is fabricated by means of thermally induced phase separation (TIPS), a novel method that can be used for the preparation of hierarchically structured monolith without any templates assisted. Three hierarchically structured adsorbents (D-PAN, H-PAN, A-PAN) based on PAN monolith were successfully prepared by reacting with diethylenetriamine (DETA), sodium hydroxide, and hydroxylamine hydrochloride, respectively. The $\mathrm{Pb}$ (II) adsorption ability of all modified samples was determined by a series of batch tests. Experimental data illustrated that all samples showed excellent $\mathrm{Pb}$ (II) adsorption ability and the maximum adsorption capacity of D-PAN, H-PAN, and A-PAN was $144 \mathrm{mg} \mathrm{g}^{-1}, 206 \mathrm{mg} \mathrm{g}^{-1}$ and $242 \mathrm{mg} \mathrm{g}^{-1}$, respectively. The feasible preparation of

\footnotetext{
${ }^{*}$ Corresponding author: Tel/Fax: +86-411-84986024. E-mail address: jqiu@dlut.edu.cn (J. Qiu)
} 


\section{Chemical Engineering Journal}

hierarchically monolithic adsorbents with high adsorption capacities opens a new expectation in the potential application for engineering.

Keywords: Adsorption; Adsorbent; Monolith; Hierarchical structure; Heavy metal

\section{Introduction}

With the huge development of productivity, water pollution caused by heavy metals, especially by $\mathrm{Pb}$ (II), has become one of the most critical environmental and public health problems[1]. Although appropriate lead intake is harmless to the human body, excess will cause serious diseases. For example, excessive lead can cause damage to liver, kidney and reduction in hemoglobin formation[2]. According to the report of the World Health Organization (WHO), the provisional tolerable weekly intake of $\mathrm{Pb}$ (II) is $0.025 \mathrm{mg} \mathrm{kg}^{-1}$ body weight for all human groups[3]. As a result, the removal and recovery of the heavy metals from aqueous solution has become one of the most essential aspects of environment research.

A number of methods, such as chemical precipitation, adsorption, capacitive deionization and liquid extraction, have been developed over the years[4-9]. Adsorption has attracted much more attention due to its high efficiency, low cost and reusability[10]. Many kinds of conventional adsorbents such as activated carbon, zeolites and clays have been used for the removal of heavy metals[11-13]. But it is difficult for conventional adsorbents to separate the adsorbents from solution, which is a necessary task[14]. To solve the separation problem, many researchers are 


\section{Chemical Engineering Journal}

resorting to grafting functional groups (e.g., amidoxime, amino and carboxyl) onto the electrospun nanofibers[15-19]. The monolith chelating fibers, easy to separate, have interconnected macropore which is helpful to the diffusion of ions[20]. These works provide an effective method for the settlement of the separation problem. However, there is only but one disadvantage that macropore alone will limit the adsorption capacity[21]. Relative studies have confirmed that the mesopore or micropore will contribute to the increase of the adsorption capacity[22-24]. Ma and co-workers have recently functionalized the Covalent Organic Frameworks (COFs) with thiol groups[23]. Due to abundant pores of the COFs, the adsorbent exhibits an exceptional mercury saturation uptake, with a capacity of over $1,000 \mathrm{mg} \mathrm{g}^{-1}$.

Hiroshi Uyama et al. provided a facile and easy technique named thermally induced phase separation (TIPS) to prepare polymer monoliths[25]. A continuous interconnected network of polyacrylonitrile (PAN) monolith consisting of macropores and mesopores can be successfully prepared. The monolith exhibits high surface area, achieved by mesopore, providing more active adsorption sites and connected macropore structures for the ions diffusion. As reported, the hierarchical structure has a good performance in the adsorption field[26]. Herein, the monoliths were grafted with different functional groups to remove heavy metals from aqueous solution. Owing to the hierarchical structure, all samples exhibited fast kinetics and the adsorption equilibrium could be reached within 3 hours. Besides, the influences of initial $\mathrm{pH}$ and ionic strength were also considered. Desorption experiments by elution of adsorbents with $1 \mathrm{M}$ hydrochloric acid showed that all samples exhibited 
outstanding reusability. The study provides direction for a hierarchically structured adsorbent design.

\section{Experimental}

\subsection{Materials}

PAN with a molecular weight of $150,000 \mathrm{~g} \mathrm{~mol}^{-1}$ and dimethylsulfoxide (DMSO) are provided by Sigma-Aldrich. Sodium hydroxide, diethylenetriamin (DETA), aluminum chloride, hydroxylamine hydrochloride $\left(\mathrm{NH}_{2} \mathrm{OH} \cdot \mathrm{HCl}\right)$ and sodium carbonate are purchased from Tianjin Reagent Chemicals. Lead nitrate of analytical grade is used. All chemical materials are used as received without further purification.

\subsubsection{Preparation of the Monolith}

The PAN monolith with a hierarchical structure was prepared by TIPS[18]. Typically raised the temperature to $373 \mathrm{~K}$, and $1 \mathrm{~g}$ PAN powder dissolved in $10 \mathrm{~mL}$ DMSO/ $\mathrm{H}_{2} \mathrm{O}$ (volume ratio $88 / 12$ ) mixture solvent. Then the polymer solution was cooled at $293 \mathrm{~K}$. The polymer would be separated out via phase separation and formed a monolith. The monolith was recovered and washed with water to remove the DMSO embedded in it. Finally the monolith was dried at a freezing dryer for one week. The general process for the synthesis of the polymer monolith was illustrated in Fig. 1. 


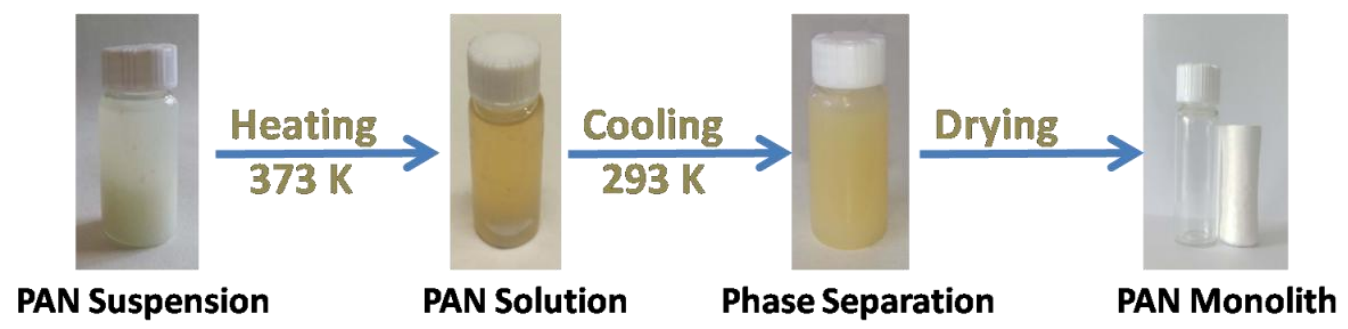

Fig. 1 Digital photographs of the general process for synthesis of the polymer.

\subsubsection{Preparation of H-PAN monolith}

The nitrile group of the PAN monolith was converted into carboxyl (labeled as H-PAN) by reacting with sodium hydroxide aqueous solution. The scheme was shown in Scheme 1. The detailed process was as follows. First, $1 \mathrm{~g}$ monolith was hydrolyzed by being immersed in $40 \mathrm{~mL} 2 \mathrm{M}$ sodium hydroxide aqueous solution under stirring at $338 \mathrm{~K}$ for 60 minutes. The color of the monolith turned red from white in this step. Then the hydrolyzed monolith was picked up from the alkaline solution and treated with $0.5 \mathrm{M} \mathrm{HCl}$ until the color turned to yellow. Finally the monolith was washed repeatedly with deionized water, and dried in a freezing dryer.

\subsubsection{Preparation of D-PAN monolith}

The DETA was grafted onto the monolith (labeled as D-PAN) through the reaction between DETA and nitrile group of the monolith. The corresponding scheme was given in Scheme 2. Typically, $0.6 \mathrm{~g}$ PAN monolith was added into $50 \mathrm{~mL}$ DETA solution containing $1.1 \mathrm{~g} \mathrm{AlCl}_{3} \cdot 6 \mathrm{H}_{2} \mathrm{O}$, the solution temperature being fixed at $353 \mathrm{~K}$ for 3 hours and with continuously stirring. The color of the product turned to faint yellow. Finally, the product was washed thoroughly by deionized water and dried in a 
freezing drier.

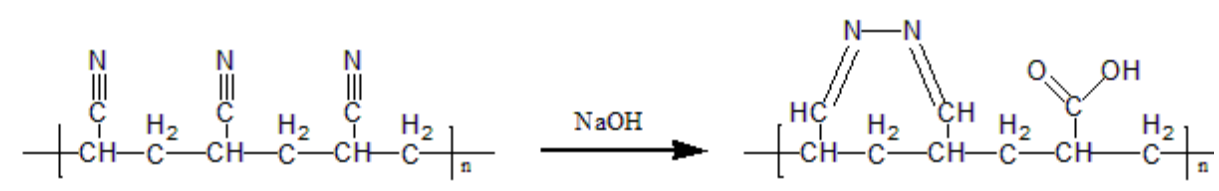

Scheme 1 The reaction process of PAN hydrolysis.

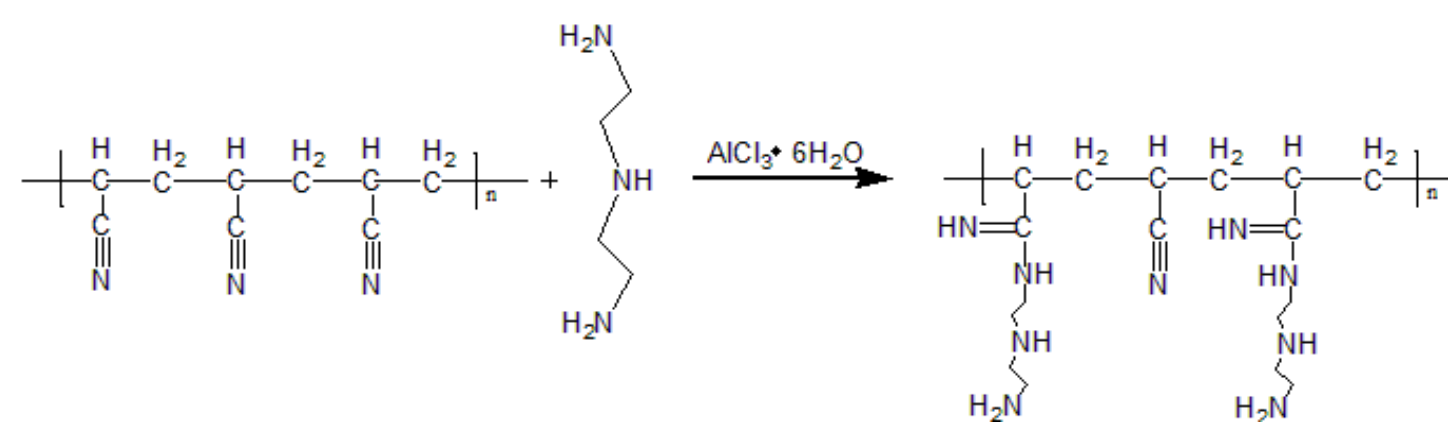

Scheme 2 The reaction process of PAN animated.

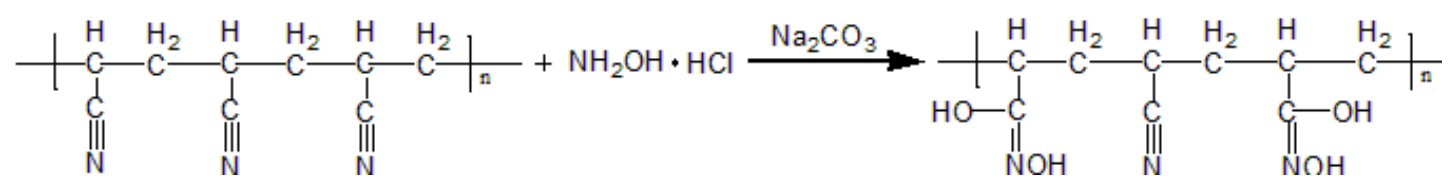

Scheme 3 The reaction process of PAN amidoxime.

\subsubsection{Preparation of A-PAN monolith}

The nitrile group of the monolith was converted into amidoxime (labeled as A-PAN) by reacting with hydroxylamine hydrochloride. The related scheme was provided in the Scheme 3. First, $1 \mathrm{~g}$ PAN monolith was immersed in $100 \mathrm{~mL}$ deionized water containing $2 \mathrm{~g}$ hydroxylamine hydrochloride. The $\mathrm{pH}$ of the solution was 
adjusted to 7 with sodium carbonate. The solution was stirred continuously with a magnetic stirrer. Then the temperature of the solution was fixed at $343 \mathrm{~K}$ for $3 \mathrm{~h}$. The color of the monolith changed from white to yellow. Finally, the product was washed several times with deionized water and dried in a freezing drier.

\subsection{Characterization}

Functional groups of adsorbents were identified with Fourier transform infrared spectrometry ( FTIR, Thermo Scientic, 6700) based on KBr method, with a resolution of $4 \mathrm{~cm}^{-1}$ and a wavenumber range of $4000-400 \mathrm{~cm}^{-1}$. Morphology of samples, was observed and recorded with scanning electron microscope ( SEM, FEI, Nova 450). Pb

(II) in the solution was determined by the Atomic Fluorescence Spectroscopy (AFS, Jitian8020). The Brunauer-Emmett-Teller (BET) nitrogen adsorption/desorption isotherms and corresponding pore size of all samples were obtained by Micromeritics ASAP 2020 at $77 \mathrm{~K}$.

\subsection{Adsorption study}

All the adsorption experiments were carried out as follows. Briefly, adsorbents were weighted and immersed in $\mathrm{Pb}$ (II) solution of different concentration with continuously stirring for a period of time which was determined by the kinetic study until reaching the adsorption equilibration. The residual $\mathrm{Pb}$ (II) in the solution was determined by AFS. All test temperatures without pointing out were $303 \mathrm{~K}$. The pH value of the testes was adjusted with $0.1 \mathrm{M} \mathrm{HCl}$ or $0.1 \mathrm{M} \mathrm{NaOH}$. The effects of initial 
$\mathrm{pH}$, the initial $\mathrm{Pb}$ (II) concentration solution at appropriate $\mathrm{pH}$ for $12 \mathrm{~h}$, the contact time and the ionic strength on adsorption were examined. The detail of the test is that $200 \mathrm{mg}$ adsorbent is added into $200 \mathrm{~mL} \mathrm{~Pb}$ (II) solution under continuous stirring and the temperature is fixed on $303 \mathrm{~K}$. Then take part of the solution at set intervals and measure its $\mathrm{Pb}$ (II) concentration.

Specially, the absorption capacity $\mathrm{Q}\left(\mathrm{mg} \mathrm{g}^{-1}\right)$ was calculated by the following equation:

$$
\begin{aligned}
& Q_{\mathrm{e}}=\frac{\left(C_{0}-C_{\mathrm{e}}\right) V}{W} \\
& Q_{\mathrm{t}}=\frac{\left(C_{0}-C_{\mathrm{t}}\right) V}{W}
\end{aligned}
$$

Where $C_{0}\left(\mathrm{mg} \mathrm{L}^{-1}\right), C_{\mathrm{t}}\left(\mathrm{mg} \mathrm{L}^{-1}\right)$ and $C_{\mathrm{e}}\left(\mathrm{mg} \mathrm{L}^{-1}\right)$ are the $\mathrm{Pb}$ (II) concentration at the time of initial, $t$ and the equilibrium respectively. $W(\mathrm{~g})$ is the weight of the adsorbents while $V(\mathrm{~L})$ is the volume of the solution.

\subsection{Desorption and regeneration study}

It was necessary to evaluate the regeneration ability of the adsorbents, since regeneration ability was an important aspect of adsorbents. Thus desorption experiments were carried out repeatedly as follows. After absorbing $\mathrm{Pb}$ (II), the adsorbents were washed with deionized water to remove the unabsorbed $\mathrm{Pb}$ (II). Then the adsorbents were immersed in $1 \mathrm{M} \mathrm{HCl}$ aqueous solution for 60 minutes, to substitute $\mathrm{Pb}$ (II) thoroughly with $\mathrm{H}^{+}$. Finally the adsorbents were washed with deionized water for several times to ensure the excess $\mathrm{H}^{+}$was removed. The regenerated adsorbents were used in the next adsorption test. The desorption 
efficiency $(E)$ is calculated as follows:

$$
E(\%)=\frac{Q_{\mathrm{e}(\mathrm{n})}}{Q_{\mathrm{e}(1)}} \times 100 \%
$$

Where $Q_{\mathrm{e}}(\mathrm{n})$ denotes the adsorption capacity of cycle $\mathrm{n}$ and $Q_{\mathrm{e}}(1)$ denotes the adsorption capacity of cycle 1 .

\section{Results and Discussion}

\subsection{Characterization of Samples}

The morphologies of the PAN monolith and the adsorbents are observed by SEM and the photographs are given in Fig. 2. The structure of the original monolith is shown in Fig. 2(a). The monolith exhibits both inter-connected macropore $(1 \mu \mathrm{m}-2$ $\mu \mathrm{m})$ and mesopore $(25 \mathrm{~nm})$, confirmed by the nitrogen adsorption/desorption isotherms latter, on the skeleton. The hierarchical structure is helpful for the heavy metal removal process. The macropore contributes to the ions diffusion and the mesopore will produce more active sites resulting in a high adsorption capacity. Morphologies of D-PAN, A-PAN and H-PAN were shown in Fig. 2 (b) , (c) and (d), respectively. The structure of all adsorbents, compared to the original monolith, is partly destructed but the basic connected macropore structure still remains. 

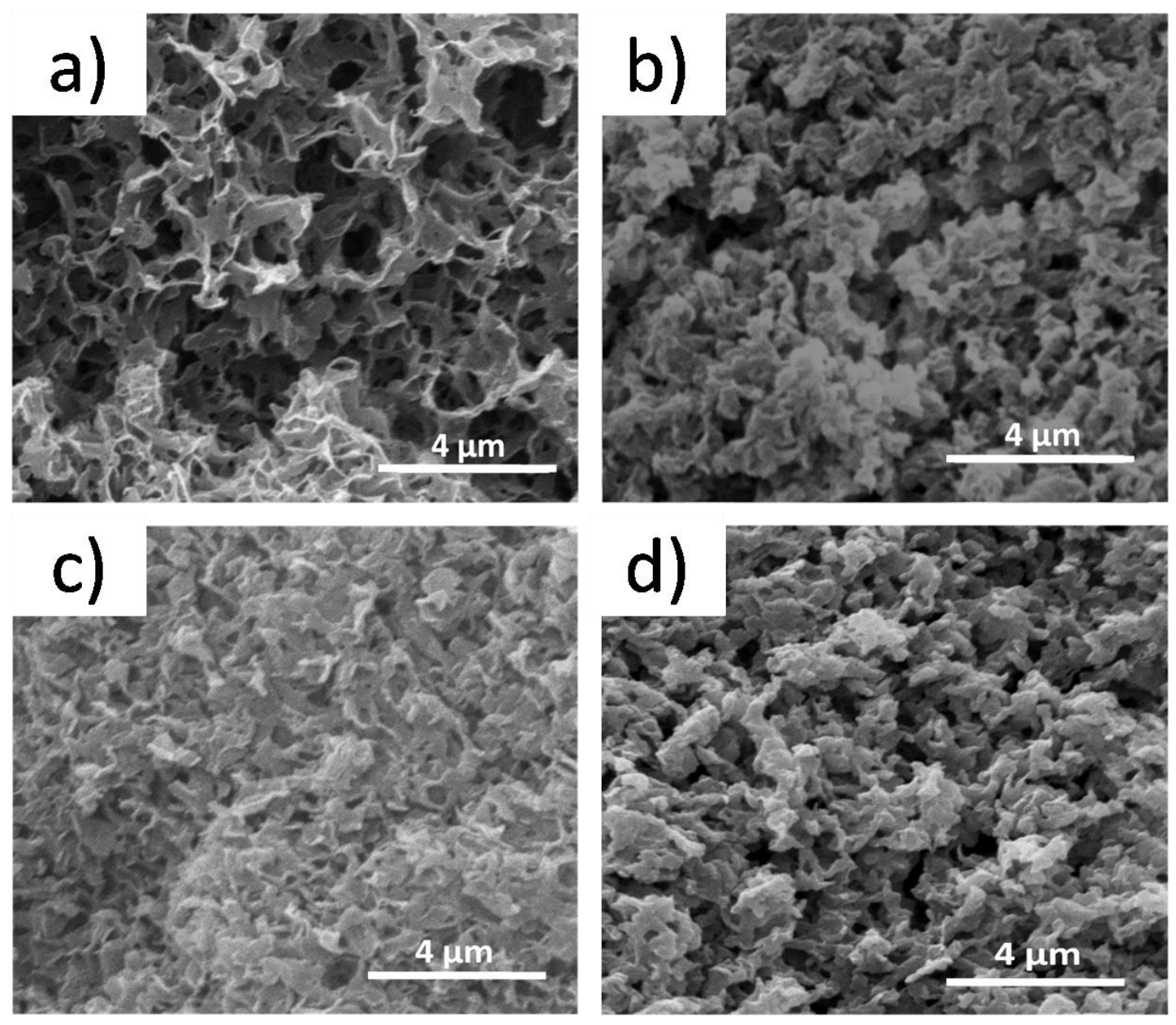

Fig. 2 The morphologies of a) PAN monolith, b) D-PAN, c) A-PAN and d) H-PAN

FTIR spectra of all samples are given in Fig. 3. The original monolith exhibits strong adsorption peak at $2240 \mathrm{~cm}^{-1}$, corresponding to the stretching vibration of nitrile group of PAN. The adsorption peaks at $2940 \mathrm{~cm}^{-1}$ and $1370 \mathrm{~cm}^{-1}$ correspond to the stretching vibration and scissoring vibration of methyl group $(\mathrm{C}-\mathrm{H})$ separately. The spectrum of H-PAN adsorbent illustrates that the strength of bands at $3346 \mathrm{~cm}^{-1}$ and $1670 \mathrm{~cm}^{-1}$ are increased, indicating the existence of hydroxyl group $(\mathrm{O}-\mathrm{H})$ and ester group $(\mathrm{C}=\mathrm{O})$. There also exhibits adsorption peaks at $1558 \mathrm{~cm}^{-1}, 1404 \mathrm{~cm}^{-1}$, $1223 \mathrm{~cm}^{-1}$, which are caused by the imine conjugated sequences $(-\mathrm{C}=\mathrm{N}-)$ [27]. 
Compared with the original PAN monolith, the spectrum of D-PAN adsorbent exhibits new adsorption peaks at $3445 \mathrm{~cm}^{-1}, 1607 \mathrm{~cm}^{-1}$ and $1490 \mathrm{~cm}^{-1}$, which corresponding to the stretching vibrations of amine group $(\mathrm{N}-\mathrm{H})$, the amidine group $(\mathrm{C}=\mathrm{N})$ and the bending vibrations of amine group $(\mathrm{N}-\mathrm{H})[28]$. In the spectrum of A-PAN, the adsorption peaks appear at $3431 \mathrm{~cm}^{-1}, 1648 \mathrm{~cm}^{-1}, 1400 \mathrm{~cm}^{-1}$ and 918 $\mathrm{cm}^{-1}$, which illustrates the existence of stretching vibrations of hydroxyl group $(\mathrm{O}-\mathrm{H})$ or amine group $(\mathrm{N}-\mathrm{H})$, amidine group $(\mathrm{C}=\mathrm{N}), \mathrm{C}-\mathrm{N}$ and $\mathrm{N}-\mathrm{O}$ respectively. According to the FTIR spectra, it is confirmed that the functional groups have been grafted on the original PAN monolith successfully[29]. The active content can be determined by FTIR using internal standard method. The methyl group will not take part in the reaction and the nitrile group is the only modified site. Thus, regarding the intensity of the methyl group's peak as constant, active contents can be determined by measuring the changing nitrile group peak's intensity in adsorbents. There are about $83 \%, 71 \%$ and $61 \%$ nitrile groups converting to active contents in the adsortents of H-PAN, D-PAN and A-PAN separately. 


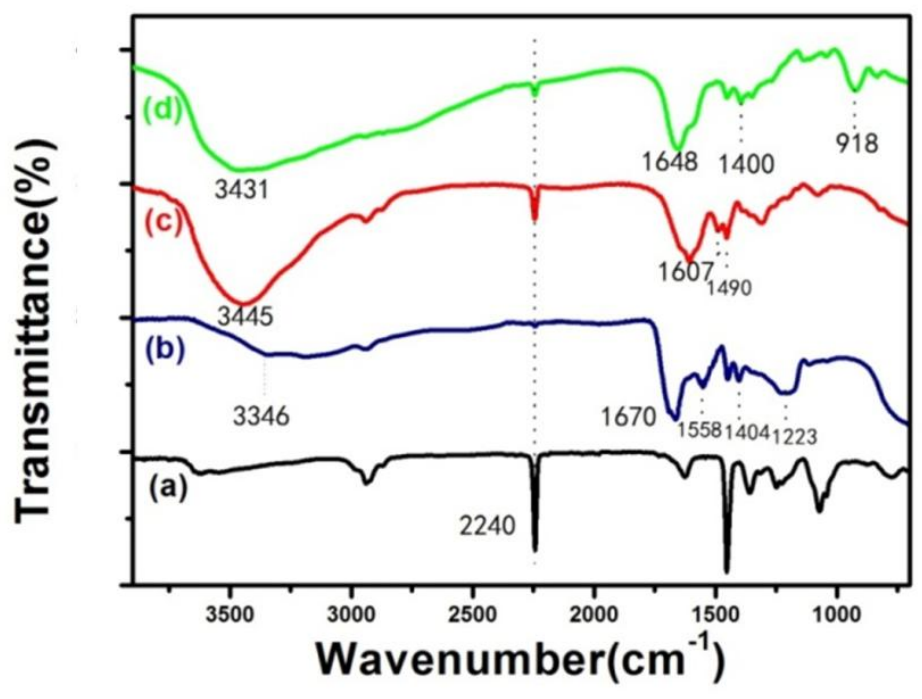

Fig.3 The FTIR spectra of all samples, (a) original PAN monolith, (b) H-PAN, (c)

$$
\text { D-PAN, ( d) A-PAN. }
$$

The nitrogen adsorption/desorption isotherms for all samples are given in Fig. 4. All samples exhibit a typical type IV isotherm with a $\mathrm{H}_{1}$ type hysteresis loop. Fig. 4 (a) is the nitrogen adsorption/desorption isotherm for the original PAN monolith. The nitrogen adsorption increases steadily in the $\mathrm{P} / \mathrm{P}_{0}$ range from 0.05 to 0.7 but begins to inflect sharply in the $\mathrm{P} / \mathrm{P}_{0}$ range from 0.7 to 1.0 , which indicates the existence of the mesopore and macropore. The specific surface area calculated from the BET equation is about $160 \mathrm{~m}^{2} \mathrm{~g}^{-1}$, which is much larger than that of the electrospun nanofiber. The pore size distribution (PSD) plot for the sample obtained by using BJH method reveals the pore diameter is around $25 \mathrm{~nm}$. The nitrogen adsorption/desorption isotherms of functionalized monoliths are given in Fig.4 (b) (H-PAN, D-PAN y-axis values offset by $25 \mathrm{~cm}^{3} \mathrm{~g}^{-1}$ and $50 \mathrm{~cm}^{3} \mathrm{~g}^{-1}$ respectively for clarity). It is clear that the maximum nitrogen adsorption amount decreases. However the shape of the isotherms 
is similar to the original monolith. The PSD is also similar to the original PAN monolith. The surface area of H-PAN, D-PAN and A-PAN is $21 \mathrm{~m}^{2} \mathrm{~g}^{-1}, 60 \mathrm{~m}^{2} \mathrm{~g}^{-1}$ and $20 \mathrm{~m}^{2} \mathrm{~g}^{-1}$, respectively. The result indicates that the structure of the monolith is partly destructed but the hierarchical structure still remains.
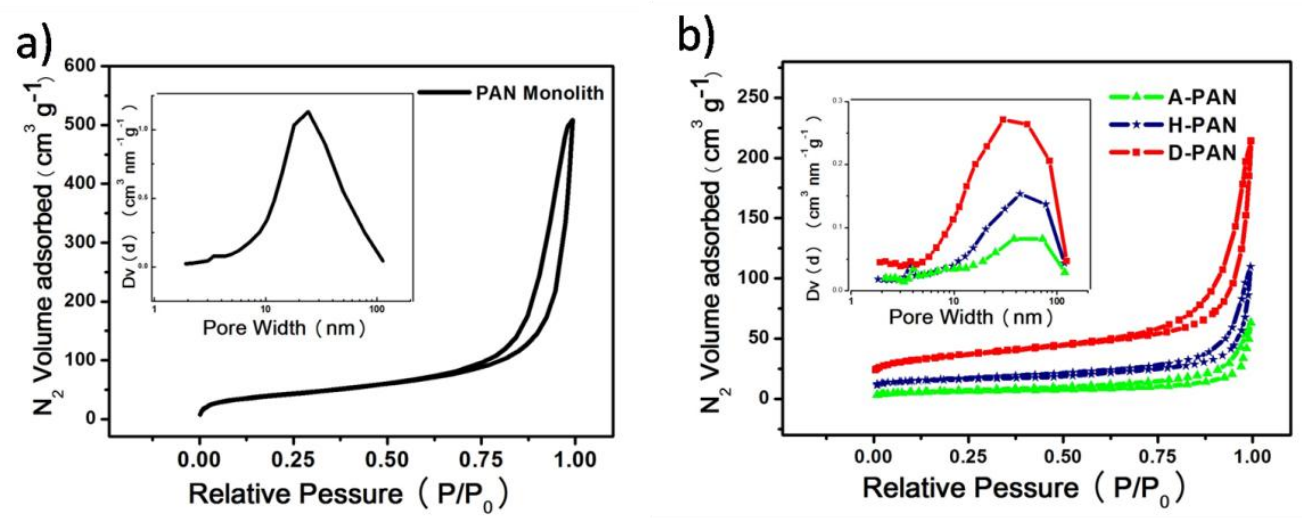

Fig.4 The nitrogen adsorption/desorption isotherms (a) the original PAN monolith, (b) the functionalized monoliths

\subsection{Adsorption of $\mathrm{Pb}$ (II) on functionalized monoliths}

\subsubsection{Effect of initial pH}

Since $\mathrm{pH}$ value of solution is an important factor for the adsorption process, all the samples will be investigated as a function of the initial $\mathrm{pH}$ in the $130 \mathrm{mg} \mathrm{L}^{-1} \mathrm{~Pb}$ (II) solution. The initial $\mathrm{pH}$ value of the $\mathrm{Pb}$ (II) solution is adjusted with $0.1 \mathrm{M} \mathrm{HCl}$ or 0.1 $\mathrm{M} \mathrm{NaOH}$. To avoid the precipitation of $\mathrm{Pb}$ (II) ions, all the experiments are carried out at $\mathrm{pH} 2-6$. The adsorption time is $12 \mathrm{~h}$ and the temperature of the whole adsorption process is fixed on $303 \mathrm{~K}$. The result was given in Fig. 5. At lower $\mathrm{pH}$, little $\mathrm{Pb}$ (II) could be adsorbed on adsorbents. The main reason is the competition between $\mathrm{H}^{+}$and $\mathrm{Pb}$ (II). As initial $\mathrm{pH}$ value increases, all samples exhibit higher adsorption capacity 
and the maximum capacity is obtained at $\mathrm{pH}=5$. Hereafter, any further increase of the $\mathrm{pH}$ value will result in a decreased adsorption capacity of the adsorbents, which can be attributed to $\mathrm{OH}^{-}$, an ion that can combine $\mathrm{Pb}$ (II) to form $\mathrm{Pb}(\mathrm{OH})^{+}$, $\mathrm{Pb}_{2}(\mathrm{OH})_{2}{ }^{2+}$, causing a reduction of the free $\mathrm{Pb}$ (II) [30]. If the $\mathrm{pH}$ value is further increased, the $\mathrm{Pb}$ (II) will convert to $\mathrm{Pb}(\mathrm{OH})_{2}$ precipitate. Thus, the following adsorption tests without special explanation are all carried out at $\mathrm{pH}=5$.

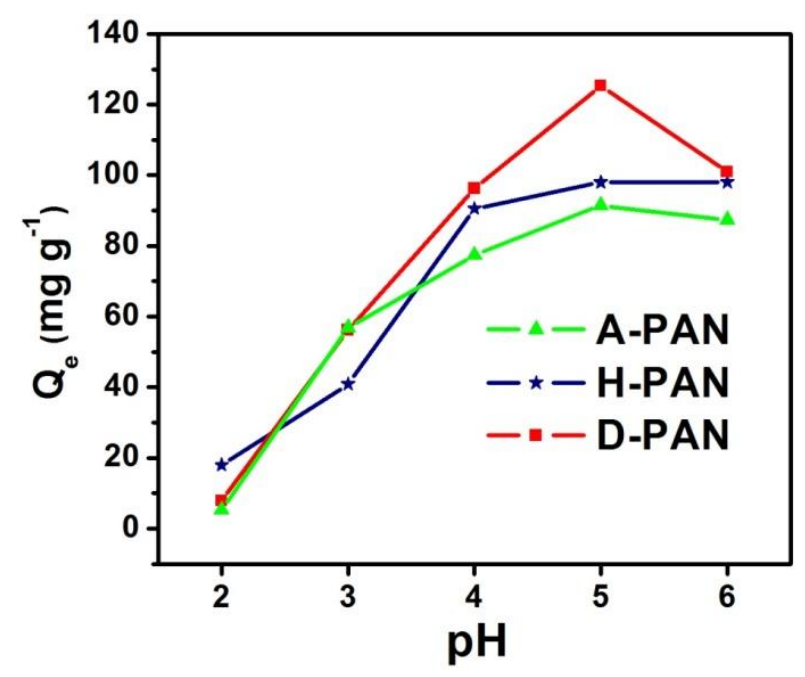

Fig. 5 The effect of initial $\mathrm{pH}$ on adsorption tests.

\subsubsection{Effect of contact time and kinetics of adsorption}

Fig. 6 shows the effect of contact time on the adsorption capacity. It is a vital factor to evaluate the performance of adsorbents. All samples were investigated in $80 \mathrm{mg} \mathrm{L}^{-1}$ $\mathrm{Pb}$ (II) solution. In order to investigate the kinetic of the adsorption process, the pseudo-first-order and pseudo-second-order kinetic model were examined. The linear formulas of these models can be described as follows. 


$$
\begin{aligned}
& \ln \left(Q_{\mathrm{e}}-Q_{\mathrm{t}}\right)=\ln Q_{\mathrm{e}}-\mathrm{k}_{1} t \\
& \frac{t}{Q_{\mathrm{t}}}=\frac{1}{\mathrm{k}_{2} Q_{\mathrm{e}}^{2}}+\frac{t}{Q_{\mathrm{e}}}
\end{aligned}
$$

Where the $\mathrm{k}_{1}\left(\mathrm{~min}^{-1}\right)$ and $\mathrm{k}_{2}\left(\mathrm{~g} \mathrm{mg}^{-1} \min ^{-1}\right)$ are the rate constants of the adsorption and $t$ is the contact time (min) in the pseudo-first-order equation (Eq. (4)) and pseudo-second-order equation (Eq. (5)).

The result shows adsorption behavior fits the pseudo-second-order model better and is shown in Fig. 6. The fitting result of pseudo-first-order model is not shown in the Fig. 6 for simplicity. But all parameters of the model are shown in Table 1. All curves are quite similar other than some differences in adsorption amount. Initially, the adsorption is swift because of the great number of free adsorptive sites and the high concentration of metal ions. In the next phase, adsorption rates decrease and finally reach equilibrium, owing to the depletion of the adsorptive sites and the decreased $\mathrm{Pb}$ (II) in the solution. Benefiting from the hierarchical structure and the formation of chelate complexes, all samples exhibit fast adsorption kinetic and reach equilibrium in 3 hours. The following tests time with no special instruction are 12 hours to ensure the fully adsorption. 


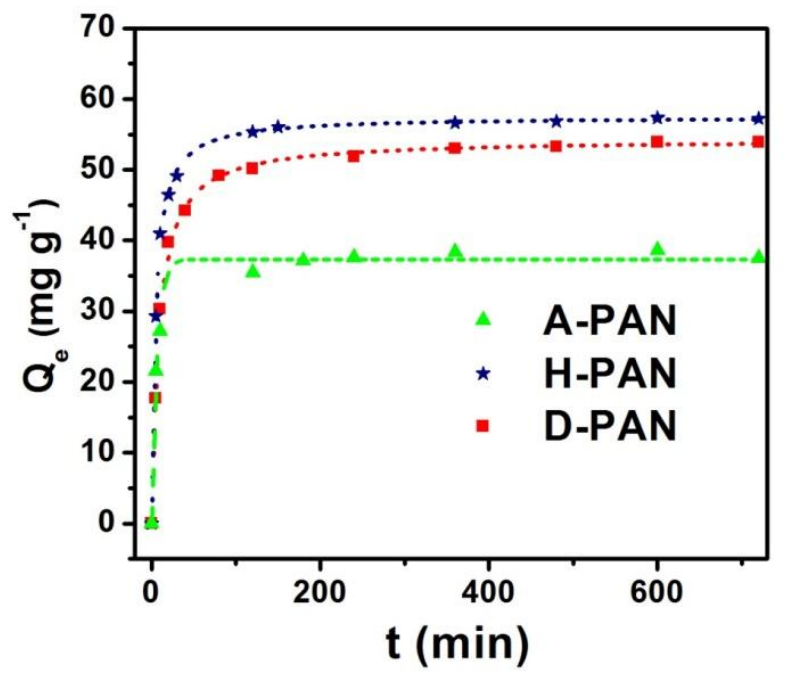

Fig.6 The effect of contact time and the kinetics of adsorption based on the pseudo-second- order kinetic model.

Table 1 Kinetics parameters describing the adsorption of $\mathrm{Pb}$ (II) onto the adsorbents, on the basis of pseudo-first-order and pseudo-second-order models.

\begin{tabular}{|c|c|c|c|c|c|c|c|}
\hline & & \multicolumn{4}{|c|}{ pseudo-first-order model } & \multicolumn{2}{|c|}{ pseudo-second-order model } \\
\hline \multirow[t]{2}{*}{ metal ion } & adsorbent & $\begin{array}{c}\mathrm{k}_{1} \\
\left(\min ^{-1}\right)\end{array}$ & $\begin{array}{r}\mathrm{q}_{\mathrm{e}, \mathrm{cal}} \\
\left(\mathrm{mg} \mathrm{g}^{-1}\right)\end{array}$ & $\mathrm{r}^{2}$ & $\mathrm{k}_{2}$ & $\begin{array}{c}\mathrm{q}_{\mathrm{e}, \mathrm{cal}} \\
\left(\mathrm{mg} \mathrm{g}^{-1}\right)\end{array}$ & $r^{2}$ \\
\hline & H-PAN & 0.016 & 50.5 & 0.720 & $2.4 \mathrm{E}-05$ & 57 & 0.998 \\
\hline \multirow[t]{2}{*}{$\mathrm{Pb}(\mathrm{II})$} & A-PAN & 0.015 & 34.1 & 0.758 & $1.5 \mathrm{E}-01$ & 37 & 0.988 \\
\hline & D-PAN & 0.027 & 45.2 & 0.535 & $5.4 \mathrm{E}-05$ & 54 & 0.996 \\
\hline
\end{tabular}




\subsubsection{Effect of initial $\mathrm{Pb}$ (II) concentration}

Initial concentration of $\mathrm{Pb}$ (II) solution has great influence on adsorption capacity. The results are shown in Fig. 7. Adsorption isotherm data are analyzed with the help of Langmuir and Freundlich isotherm models. These two models can be written as follows:

$$
\begin{aligned}
& \frac{C_{\mathrm{e}}}{Q_{\mathrm{e}}}=\frac{C_{\mathrm{e}}}{Q_{\mathrm{m}}}+\frac{1}{\mathrm{~K}_{\mathrm{L}} Q_{\mathrm{m}}} \\
& Q_{\mathrm{e}}=\mathrm{K}_{\mathrm{F}} C_{\mathrm{e}}^{1 / \mathrm{n}}
\end{aligned}
$$

Where $Q_{\mathrm{m}}\left(\mathrm{mg} \cdot \mathrm{g}^{-1}\right)$ represents the saturation uptake capacity of the metal ions on the adsorbent and $K_{\mathrm{L}}\left(\mathrm{L} \mathrm{mg}^{-1}\right)$ is the adsorption equilibrium constant. $K_{\mathrm{F}}$ and $\mathrm{n}$ are Freundlich constants. By plotting $\log Q_{\mathrm{e}}$ as a function of $\log C_{\mathrm{e}}$, the value of $K_{\mathrm{F}}$ is taken as the antilogarithmic value of the $y$-intercept and $n$ is the inverse value of the slope.

The results are reported as the functions of the adsorption amount of the adsorbent $\left(Q_{\mathrm{e}}\right)$ versus the concentration of $\mathrm{Pb}$ (II) in the solution $\left(C_{\mathrm{e}}\right)$ and all samples are fitted with Langmuir and Freundlich models. Judging by the correlation coefficient $r^{2}$, the adsorption process is better to be described with Langmuir model suggesting the adsorption of $\mathrm{Pb}$ (II) is a homogeneous and monolayer adsorption process. Considering that Langmuir model fits the data better, we only show the Langmuir fitting curve in Fig. 7, yet with the parameters of both models shown in Table 2. As shown in Table 2, using the Langmuir model, the maximum adsorption amounts for $\mathrm{Pb}$ (II) is $206 \mathrm{mg} \mathrm{g}^{-1}, 242 \mathrm{mg} \mathrm{g}^{-1}$ and $144 \mathrm{mg} \mathrm{g}^{-1}$ of H-PAN, A-PAN and D-PAN, respectively. The saturation uptake capacities of all samples in this study are superior 
due to the presence of mesopore that will provide more active sites than other traditional adsorption materials.

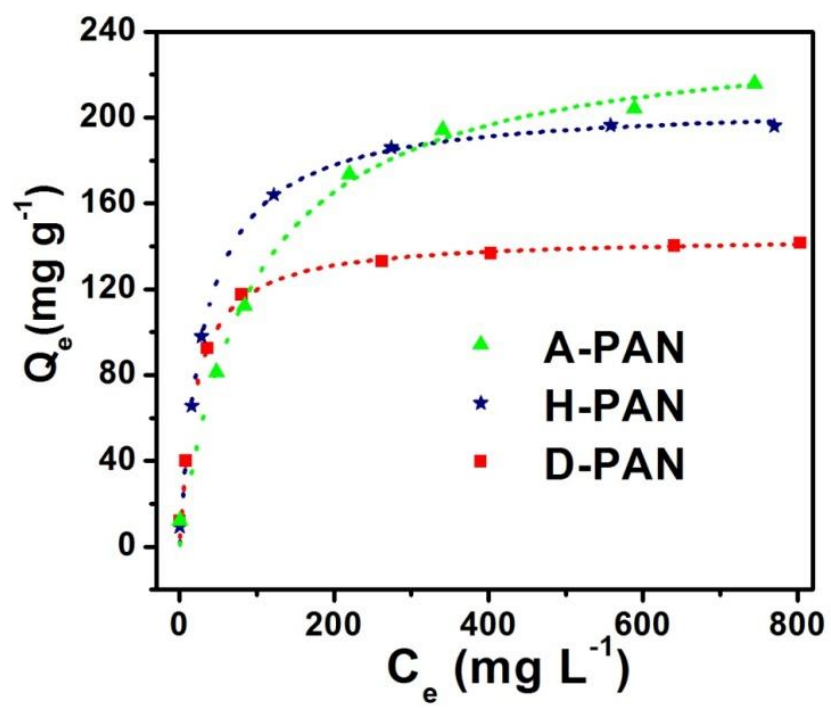

Fig. 7 The effect of initial concentration and the adsorption isotherm based on the Langmuir model.

Table 2 Adsorption isotherms parameters describing the adsorption of $\mathrm{Pb}$ (II) onto the adsorbents, on the basis of Langmuir and Freundlich models.

\begin{tabular}{|c|c|c|c|c|c|c|c|}
\hline & \multicolumn{4}{|c|}{ Langmuir model } & \multicolumn{3}{|c|}{ Freundlich model } \\
\hline \multirow[t]{2}{*}{ metal ion } & adsorbent & $\begin{array}{c}\mathrm{q}_{\mathrm{m}} \\
\left(\mathrm{mg} \mathrm{g}^{-1}\right)\end{array}$ & $\begin{array}{c}\mathrm{K}_{\mathrm{L}} \\
\left(\mathrm{L} \mathrm{mg}^{-1}\right)\end{array}$ & $r^{2}$ & $\mathrm{~K}_{\mathrm{F}}$ & $\mathrm{n}$ & $r^{2}$ \\
\hline & H-PAN & 206 & 0.03094 & 0.997 & 42.43 & 4.08 & 0.921 \\
\hline \multirow[t]{2}{*}{$\mathrm{Pb}(\mathrm{II})$} & A-PAN & 242 & 0.01073 & 0.993 & 28.58 & 3.19 & 0.964 \\
\hline & D-PAN & 144 & 0.04861 & 0.996 & 36.39 & 4.61 & 0.874 \\
\hline
\end{tabular}




\subsubsection{Effect of ionic strength}

Adsorption process will be affected by ionic strength which can be adjusted by adding sodium chloride into the solution. Certain amount of sodium chloride is added into $80 \mathrm{mg} \mathrm{L}^{-1} \mathrm{~Pb}$ (II) solution and the $\mathrm{Pb}$ (II) adsorption amounts for all samples are determined. The results of all samples are given in Fig. 8. It can be observed that the adsorption amount of H-PAN decreased with adding sodium chloride. This can be explained by the theory that the electrolyte in the solution may also compete with the heavy metal ions during the adsorption process. However, the adsorption amounts of D-PAN and A-PAN increased in the same situation. According to the theory developed by Guoy-Chapman, ions in the solution will be surrounded by the electrical diffused double layer, and an addition of electrolytes, such as sodium chloride, will significantly compress the thickness of that double layer[31]. And the change of the thickness may promote the electrostatic interaction between the adsorbents and $\mathrm{Pb}$ (II), which will cause an increase of the adsorption amount. On the other hand, adsorbent selectivity is an important parameter in evaluation of adsorbent performance. This result also show competitive adsorption results of sodium and lead ions on all adsorbents from the binary metal species solutions. All adsorbents exhibit high adsorption capacity with no sodium ion in the solution. As the concentration of sodium ions increase, however, the adsorption amount of lead ions is still relatively stable. As a result, lead ions are selectively adsorbed and separated from sodium ions in the binary metal species system. This phenomenon may be due to the difference between $\mathrm{Na}-\mathrm{N}(\mathrm{Na}-\mathrm{O})$ bond and $\mathrm{Pb}-\mathrm{N}(\mathrm{Pb}-\mathrm{O})$. The bond of $\mathrm{Pb}-\mathrm{N}(\mathrm{Pb}-\mathrm{O})$ has a high 
stability constant than that of $\mathrm{Na}-\mathrm{N}(\mathrm{Na}-\mathrm{O})$. The ligands of all adsorbents have strong affinity towards lead ions. Thus all adsorbents have a high selectivity for lead ions removal[32].

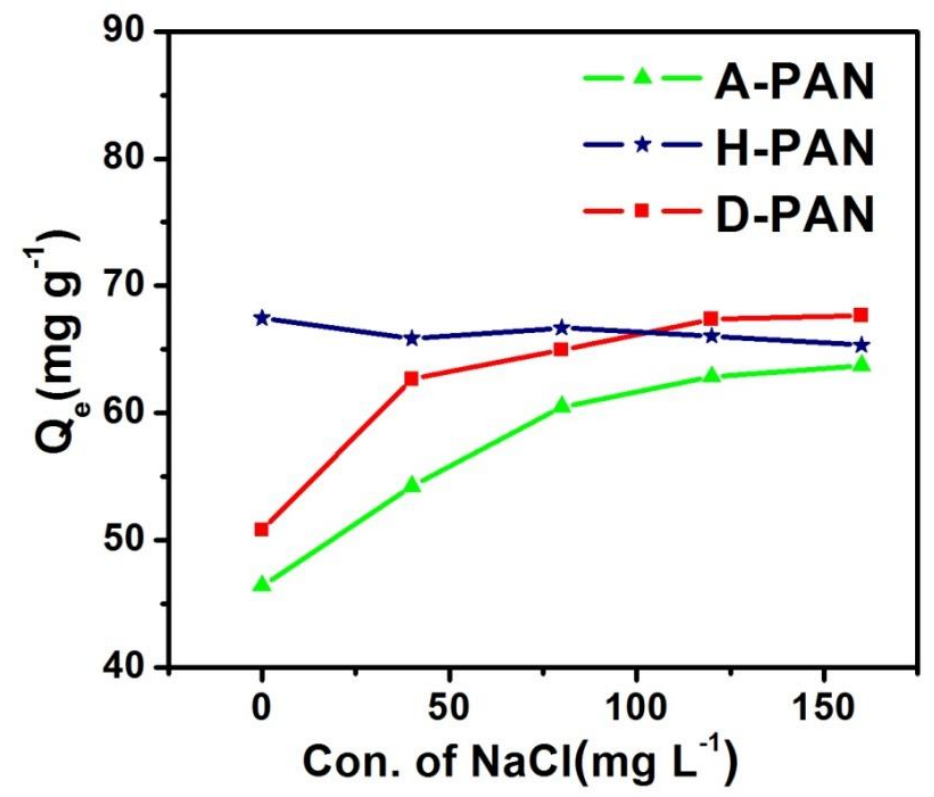

Fig. 8 The effect of ionic strength on the adsorption tests.

\subsection{Desorption study}

In order to industrialize the adsorbents, it is necessary to evaluate the regeneration ability of the adsorbents in the adsorption process. Thus, sorption-desorption cycles are performed four times to examine the reusability of all adsorbents. In this study, 1 M HCL solution was used to desorb the $\mathrm{Pb}$ (II), and desorption efficiency for the four cycles is summarized in Fig. 9. It can be seen that adsorption amounts of $\mathrm{Pb}$ (II) by all three samples decrease slightly as the regeneration cycles progress, indicating that 1 M HCL solution can effectively desorb $\mathrm{Pb}$ (II) from all adsorbents. Although the three samples manifest different reusability during the cycles, the worst of them still 


\section{Chemical Engineering Journal}

retained more than $70 \%$ of its original adsorption capacity. The results illustrate that the three samples are expected to be the promising adsorbents in the engineering field.

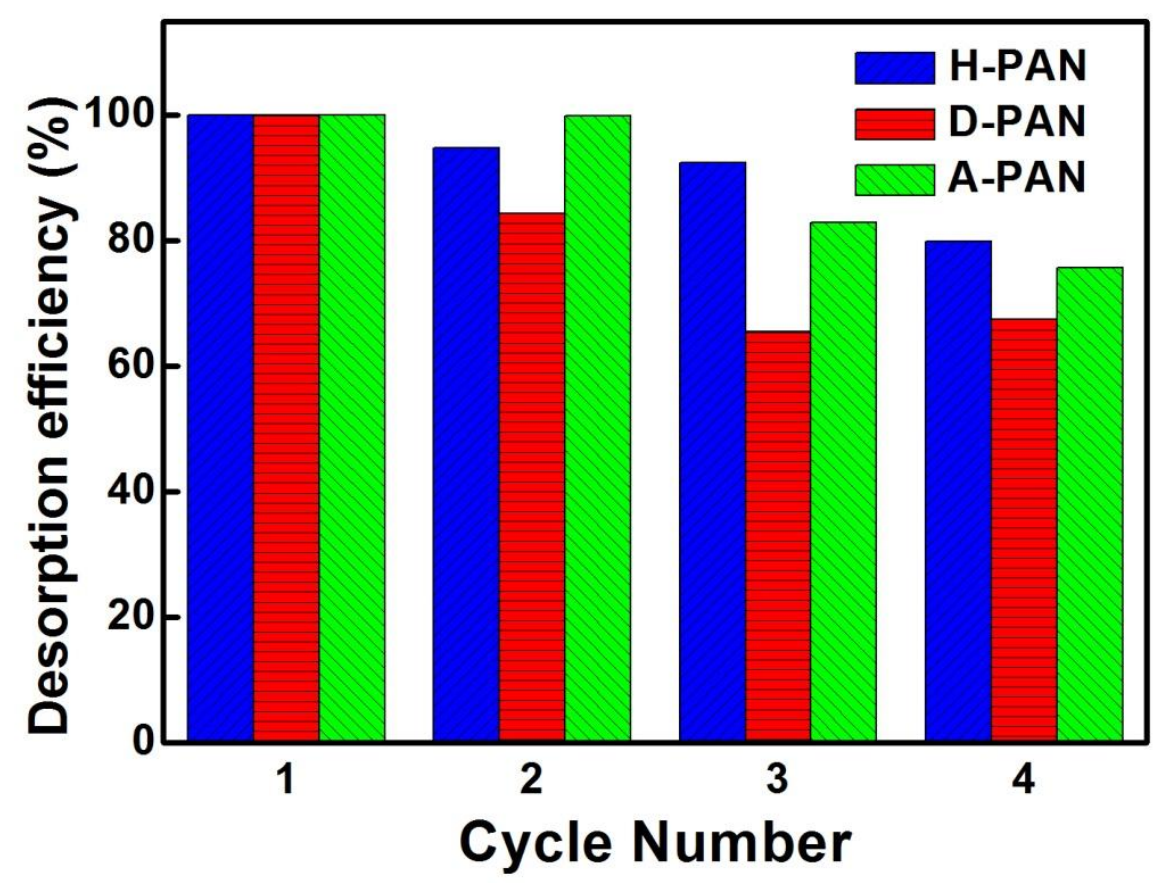

Fig. 9 The regeneration tests of all samples.

\subsection{Comparison with other adsorbents}

In comparison with the similarly reported adsorbents, listed in Table 3, the adsorbents prepared in this work show a huge advantage. First, the monolith adsorbents are easy to scale-up. PAN is a type of low cost material and has already achieved industrial production. Besides, the preparation process is not complicated and the monolithic adsorbents can be fabricated simply by controlling the temperature. Second, the saturation uptake capacities of H-PAN, D-PAN, A-PAN are much higher than the adsorbents functionalized with similar functional groups, which can attribute 
to the presence of hierarchical structure and suitable functional groups. Third, compared with the powder adsorption material, the monolithic matrix can easy to be separated and it can be made into any shape by TIPS to suit the adsorption column, which will have significant effects on the engineering operating.

Table 3 Comparison with other reported adsorbents.

\begin{tabular}{|c|c|c|}
\hline Adsorbents & $\begin{array}{c}\text { Adsorption } \\
\text { capacity }(\mathrm{mg} / \mathrm{g}\end{array}$ & Reference \\
\hline Chitosan nanofibri & 117 & {$[33]$} \\
\hline Chitosan linked with glutaraldehyde & 113 & {$[34]$} \\
\hline Salicylic acid type chelate adsorbent & 86 & {$[35]$} \\
\hline $\mathrm{Fe}_{3} \mathrm{O}_{4} / \mathrm{MnO}_{2}$ & 53 & {$[36]$} \\
\hline P-Polyacrylonitrile nanofiber & 98 & [37] \\
\hline PU@PDA@MSNs sponge & 104 & {$[38]$} \\
\hline $\mathrm{NH}_{2}-\mathrm{MCM}-41$ & 58 & [39] \\
\hline Magnetic composite & 61 & {$[40]$} \\
\hline $\mathrm{H}-\mathrm{PAN}$ & 206 & This work \\
\hline D-PAN & 144 & This work \\
\hline A-PAN & 242 & This work \\
\hline
\end{tabular}

\section{Conclusion}

In the study, the hierarchically structured polyacrylonitrile monolith is prepared by 


\section{Chemical Engineering Journal}

TIPS, which can be proved by scanning electron microscope (SEM) and nitrogen adsorption/desorption isotherms. The FTIR spectroscopy indicates that the monolith has been grafted with different functional groups successfully through a series of reactions, just as expected. And the functionalized monoliths exhibit excellent ability for removal of $\mathrm{Pb}$ (II) from aqueous solution. The maximum adsorption capacity of D-PAN, H-PAN and A-PAN is $144 \mathrm{mg} \mathrm{g}^{-1}, 206 \mathrm{mg} \mathrm{g}^{-1}$ and $242 \mathrm{mg} \mathrm{g}^{-1}$, respectively and their adsorption behaviors fit the Langmuir model better. Other factors that influence the adsorption process, such as $\mathrm{pH}$, contact time, initial concentration, ionic strength, are also considered. It is proved that $\mathrm{pH}=5$ is the optimum condition for all samples to remove $\mathrm{Pb}$ (II) from aqueous solution. The adsorption process of all adsorbents reach adsorption equilibrium in 3 hours and fit the pseudo-second-order model better. The ionic strength also has an influence on the adsorption of $\mathrm{Pb}$ (II). Furthermore as-made adsorbents exhibit good regeneration ability. After 4 cycles, the adsorption ability of all samples still maintains over $70 \%$. Therefore, it can be concluded that the functionalized monoliths are promising adsorbents for the removal of heavy metal ions from aqueous solution.

\section{Acknowledgements:}

The authors acknowledge financial support from the Qaidam Salt Lake Chemical Joint Research Fund Project of NSFC and Qinghai Province State People's Government (No. U1507103), the National Science Foundation of China (No. 21336001), the Star of the Youth Science and Technology of Dalian (No. 2015R053) 


\section{Chemical Engineering Journal}

and the Fundamental Research Funds for the Central Universities (No. DUT16TD14).

\section{References:}

[1] Y. Deng, Z. Gao, B. Liu, X. Hu, Z. Wei, C. Sun, Selective removal of lead from aqueous solutions by ethylenediamine-modified attapulgite, Chem Eng J, 223 (2013) 91-98.

[2] A.K. Meena, G.K. Mishra, P.K. Rai, C. Rajagopal, P.N. Nagar, Removal of heavy metal ions from aqueous solutions using carbon aerogel as an adsorbent, J Hazard Mater, 122 (2005) 161-170.

[3] P. Liang, H. Sang, Determination of trace lead in biological and water samples with dispersive liquid-liquid microextraction preconcentration, ANAL BIOCHEM, 380 (2008) 21-25.

[4] F. Fu, L. Xie, B. Tang, Q. Wang, S. Jiang, Application of a novel strategy-Advanced Fenton-chemical precipitation to the treatment of strong stability chelated heavy metal containing wastewater, Chem Eng J, 189-190 (2012) 283-287.

[5] C. Bertagnolli, A. Grishin, T. Vincent, E. Guibal, Recovering Heavy Metal Ions from Complex Solutions Using Polyethylenimine Derivatives Encapsulated in Alginate Matrix, Ind. Eng. Chem. Res, 55 (2016) 2461-2470.

[6] H.X. Lu, J.Y. Wang, S.F. Bu, M.D. Zhang, J.B. Zhang, Removal of nickel ions from dilute heavy metal solutions by electrodeionization process, Environmental Biotechnology And Materials Engineering, Pts 1-3, 183-185 (2011) 580-584.

[7] G.-T. Wei, Z. Yang, C.-J. Chen, Room temperature ionic liquid as a novel medium for liquid/liquid extraction of metal ions, Anal Chim Acta, 488 (2003) 183-192.

[8] X. Chen, K.F. Lam, S.F. Mak, K.L. Yeung, Precious metal recovery by selective adsorption using biosorbents, J Hazard Mater, 186 (2011) 902-910.

[9] X. Chen, K.F. Lam, K.L. Yeung, Selective removal of chromium from different aqueous systems using magnetic MCM-41 nanosorbents, Chem Eng J, 172 (2011) 728-734.

[10] F. Ke, L.G. Qiu, Y.P. Yuan, F.M. Peng, X. Jiang, A.J. Xie, Y.H. Shen, J.F. Zhu, Thiol-functionalization of metal-organic framework by a facile coordination-based postsynthetic strategy and enhanced removal of Hg2 + from water, J Hazard Mater, 196 (2011) 36-43.

[11] K.A.K.a.T.S. Anirudhan*, Uptake of Heavy Metals in Batch Systems by Sulfurized Steam Activated Carbon Prepared from Sugarcane Bagasse Pith, Ind. Eng. Chem. Res., 41 (2002) 5085-5093.

[12] K.R. Kumrić, A.B. Đukić, T.M. Trtić-Petrović, N.S. Vukelić, Z. Stojanović, J.D. Grbović Novaković, L.L. Matović, Simultaneous Removal of Divalent Heavy Metals from Aqueous Solutions Using Raw and Mechanochemically Treated Interstratified Montmorillonite/Kaolinite Clay, Ind. Eng. Chem. Res, 52 (2013) 7930-7939.

[13] P. Demirçivi, G. Nasün-Saygili, Removal of boron from waste waters using HDTMA-modified zeolites, Desalin Water Treat, 23 (2012) 110-117.

[14] J.Q. Wang, C. Luo, G.G. Qi, K. Pan, B. Cao, Mechanism study of selective heavy metal ion removal with polypyrrole-functionalized polyacrylonitrile nanofiber mats, Appl Surf Sci, 316 (2014) 245-250.

[15] M. Abdouss, A.M. Shoushtari, A.M. Simakani, S. Akbari, A. Haji, Citric acid-modified acrylic micro and nanofibers for removal of heavy metal ions from aqueous media, Desalin Water Treat, 52 (2014) 


\section{Chemical Engineering Journal}

7133-7142.

[16] M. Shojaei, S. Sadjadi, M. Rajabi-Hamane, S.J. Ahmadi, Synthesis of TiO2/polyacrylonitrile nanofibers composite and its application to lead ions removal from waste waters, Desalin Water Treat, 56 (2014) 1403-1412.

[17] S. Deng, Bai, J.P. Chen, Aminated Polyacrylonitrile Fibers for Lead and Copper Removal, Langmuir, 19 (2003) 5058-5064.

[18] C. Luo, J. Wang, P. Jia, Y. Liu, J. An, B. Cao, K. Pan, Hierarchically structured polyacrylonitrile nanofiber mat as highly efficient lead adsorbent for water treatment, Chem Eng J, 262 (2015) 775-784. [19] P. Kampalanonwat, P. Supaphol, Preparation and Adsorption Behavior of Aminated Electrospun Polyacrylonitrile Nanofiber Mats for Heavy Metal Ion Removal, Acs Appl Mater Inter, 2 (2010) 3619-3627.

[20] H. Liu, C.-Y. Cao, F.-F. Wei, P.-P. Huang, Y.-B. Sun, L. Jiang, W.-G. Song, Flexible macroporous carbon nanofiber film with high oil adsorption capacity, J Mater Chem A, 2 (2014) 3557.

[21] H.T. Chiu, Fabrication of electrospun polyacrylonitrile ion-exchange membranes for application in lysozyme adsorption, Express Polym Lett, 5 (2011) 308-317.

[22] M.P.S. Zhonghua Hu, Mesoporous high-surface-area actived carbon, Micropor Mesopor Mat, 43 (2001) 267-275.

[23] B. Li, Y. Zhang, D. Ma, Z. Shi, S. Ma, Mercury nano-trap for effective and efficient removal of mercury(II) from aqueous solution, Nature communications, 5 (2014) 5537.

[24] J. Xiong, W. Zhu, H. Li, L. Yang, Y. Chao, P. Wu, S. Xun, W. Jiang, M. Zhang, H. Li, Carbon-doped porous boron nitride: metal-free adsorbents for sulfur removal from fuels, J. Mater. Chem. A, 3 (2015) 12738-12747.

[25] K. Okada, M. Nandi, J. Maruyama, T. Oka, T. Tsujimoto, K. Kondoh, H. Uyama, Fabrication of mesoporous polymer monolith: a template-free approach, Chem Commun, 47 (2011) 7422-7424.

[26] J.S. Hu, L.S. Zhong, W.G. Song, L.J. Wan, Synthesis of hierarchically structured metal oxides and their application in heavy metal ion removal, Adv Mater, 20 (2008) 2977-2982.

[27] P. Kampalanonwat, P. Supaphol, Preparation of Hydrolyzed Electrospun Polyacrylonitrile Fiber Mats as Chelating Substrates: A Case Study on Copper(II) lons, Ind. Eng. Chem. Res, 50 (2011) 11912-11921.

[28] P. Kampalanonwat, P. Supaphol, The Study of Competitive Adsorption of Heavy Metal Ions from Aqueous Solution by Aminated Polyacrylonitrile Nanofiber Mats, Energy Procedia, 56 (2014) 142-151.

[29] H. Zhao, X. Liu, M. Yu, Z. Wang, B. Zhang, H. Ma, M. Wang, J. Li, A Study on the Degree of Amidoximation of Polyacrylonitrile Fibers and Its Effect on Their Capacity to Adsorb Uranyl lons, Ind. Eng. Chem. Res, 54 (2015) 3101-3106.

[30] L. Tong, J. Chen, Y. Yuan, Z. Cui, M. Ran, Q. Zhang, J. Bu, F. Yang, X. Su, H. Xu, A novel lead ion-imprinted chelating nanofiber: Preparation, characterization, and performance evaluation, J Appl Polym Sci, 132 (2015).

[31] G. Kiani, M. Soltanzadeh, High capacity removal of silver(I) and lead(II) ions by modified polyacrylonitrile from aqueous solutions, Desalin Water Treat, 52 (2013) 3206-3218.

[32] C. Liu, R. Bai, Q. San Ly, Selective removal of copper and lead ions by diethylenetriamine-functionalized adsorbent: behaviors and mechanisms, Water Res, 42 (2008) 1511-1522.

[33] D. Liu, Z. Li, Y. Zhu, Z. Li, R. Kumar, Recycled chitosan nanofibril as an effective $\mathrm{Cu}(\mathrm{II}), \mathrm{Pb}(\mathrm{II})$ and Cd(II) ionic chelating agent: adsorption and desorption performance, Carbohydr Polym, 111 (2014) 


\section{Chemical Engineering Journal}

469-476.

[34] G.Z. Kyzas, P.I. Siafaka, D.A. Lambropoulou, N.K. Lazaridis, D.N. Bikiaris, Poly(itaconic acid)-grafted chitosan adsorbents with different cross-linking for $\mathrm{Pb}(\mathrm{II})$ and $\mathrm{Cd}(\mathrm{II})$ uptake, Langmuir, 30 (2014) 120-131.

[35] F.Q. An, B.J. Gao, X. Dai, M. Wang, X.H. Wang, Efficient removal of heavy metal ions from aqueous solution using salicylic acid type chelate adsorbent, J Hazard Mater, 192 (2011) 956-962.

[36] E.J. Kim, C.S. Lee, Y.Y. Chang, Y.S. Chang, Hierarchically Structured Manganese Oxide-Coated Magnetic Nanocomposites for the Efficient Removal of Heavy Metal Ions from Aqueous Systems, Acs Appl Mater Inter, 5 (2013) 9628-9634.

[37] R. Zhao, X. Li, B.L. Sun, M.Q. Shen, X.C. Tan, Y. Ding, Z.Q. Jiang, C. Wang, Preparation of phosphorylated polyacrylonitrile-based nanofiber mat and its application for heavy metal ion removal, Chem Eng J, 268 (2015) 290-299.

[38] S. Liu, J. Pan, J. Cao, X. Dai, M. Meng, R. Wu, J. Yao, Y. Yan, Simultaneous removal of Pb(II) and 2,4,6-trichlorophenol by a hierarchical porous PU@PDA@MSNs sponge with reversible "shape memory" effect, Chem Eng J, 284 (2016) 10-20.

[39] A. Heidari, H. Younesi, Z. Mehraban, Removal of $\mathrm{Ni}(\mathrm{II}), \mathrm{Cd}(\mathrm{II})$, and $\mathrm{Pb}(\mathrm{II})$ from a ternary aqueous solution by amino functionalized mesoporous and nano mesoporous silica, Chem Eng J, 153 (2009) 70-79.

[40] H. Alijani, M.H. Beyki, Z. Shariatinia, M. Bayat, F. Shemirani, A new approach for one step synthesis of magnetic carbon nanotubes/diatomite earth composite by chemical vapor deposition method: Application for removal of lead ions, Chem Eng J, 253 (2014) 456-463. 


\section{Graphical abstract}

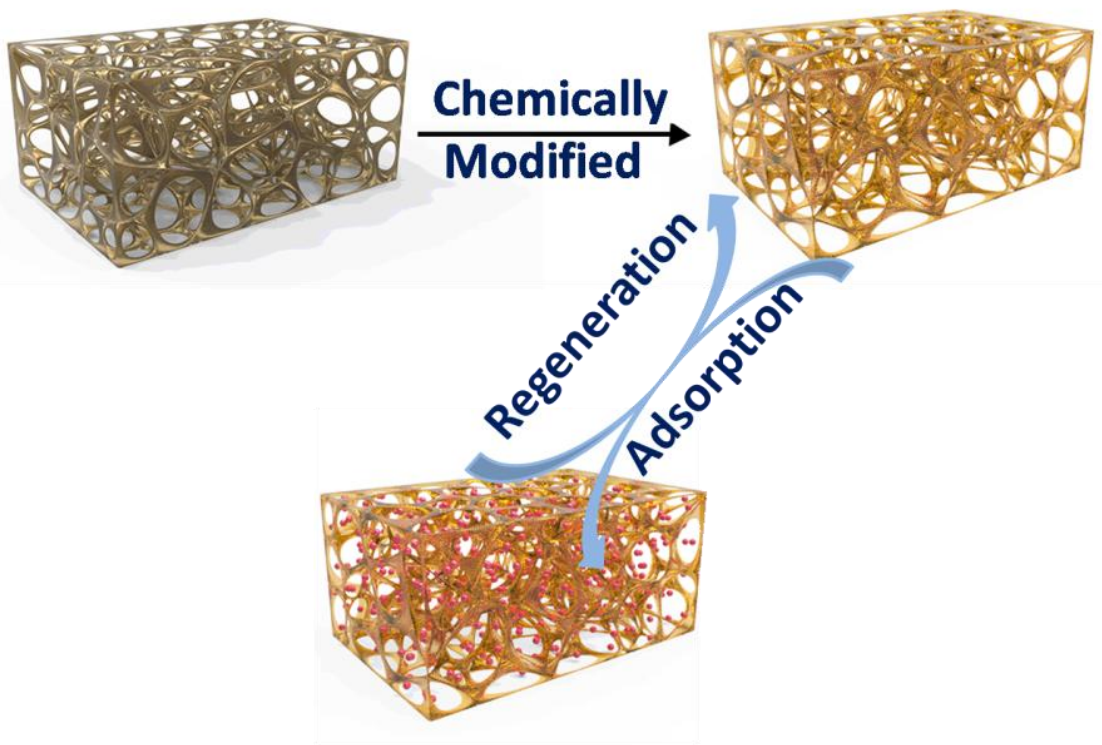

